SCIENTIFIC LETTER

\title{
Mortality caused by acute myocardial infarction in Qatari women
}

\author{
J Al Suwaidi, A Bener, S Behair, H A Al Binali
}

Heart 2004;90:693-694. doi: 10.1136/hrt.2003.014746

$\mathrm{T}$ he long held belief that coronary heart disease (CHD) in women has a more benign prognosis than in men has resulted in less aggressive diagnosis and management patterns. Contrary to this belief, CHD is the leading cause of death in white women aged 60 years or older in the USA and outnumbers the next 16 causes of death in women combined, including cancers. Furthermore, since 1980, death from CHD has declined dramatically in men in the western world but has increased in women.

With the recent recognition of this problem, considerable interest has been focused on the study of sex based differences in the outcome of patients with acute myocardial infarction (AMI) in the western world. Several studies have compared the outcome of women and men with AMI, with conflicting data. ${ }^{2-5}$ The majority of these studies have been performed in western populations and so may not necessarily apply to other ethnic groups such as the Middle Eastern population. The aim of the present study was to determine the clinical characteristics and in-hospital mortality from AMI in Qatari women.

\section{METHODS}

This study was based at Hamad General Hospital, Doha, Qatar, which is the only tertiary care centre in the country and so all patients with AMI are treated here. The Hamad General Hospital cardiology database was used for this study; this database comprises data collected from all patients admitted to the cardiology department at the hospital since January 1991. Data were collected from the clinical records by the patients' physicians at the time of the patients' hospital discharge according to predefined criteria for each data point. We focused our study only on Qatari patients because it is a stable population and avoids the bias in the fluctuation of the expatriate population in the country. The study was approved by the institution review board.

With the described database, all patients presenting with AMI in the period between 1991 and 2001 were identified. Age, sex, risk factors including smoking, hypertension, hypercholesterolaemia, diabetes, pre-existing coronary heart disease, in-hospital mortality and morbidity, as well as acute medical care provided were analysed. Data are expressed as mean (SD). Student's $t$ test was employed for comparison between the mean values of the two groups and $\chi^{2}$ analysis was performed to test for differences in proportions of categorical variables between two or more groups. Multivariate stepwise regression analysis was used to identify predictors of outcome. A probability value of $\mathrm{p}<0.05$ was considered significant.

\section{RESULTS}

During the 10 year period a total of 20856 patients were admitted to the coronary care unit and cardiology wards. Of these, 1598 Qatari patients were admitted with AMI; 1147 were male and 451 were female. When compared to men, women were older (62.1 (11.5) years $v 59.9$ (12.6) years,

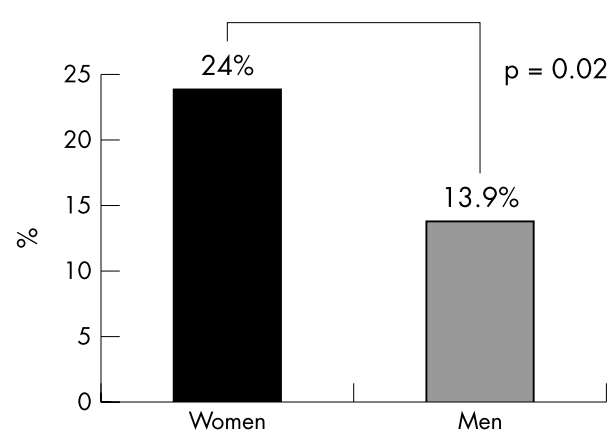

Figure 1 In-hospital mortality (\%) as a consequence of acute myocardial infarction: women versus men.

$\mathrm{p}<0.001)$ and were more likely to have diabetes $(68.5 \% v$ $48.3 \%$, p < 0.001), hypertension $(54.8 \%$ v 30.9\%, p < 0.001$)$, and hyperlipidaemia $(30.4 \% \vee 24.9 \%, \mathrm{p}=0.029)$, but were less likely to be smokers $(0.9 \% v 32.3 \%, \mathrm{p}<0.001)$. Of the women included in the study $90.9 \%$ were postmenopausal.

Thrombolytic treatment was used less often in women than in men ( $19.5 \% v 28.4 \%, p=0.08)$. $\beta$ Blockers were also used less often $(28.1 \% \vee 18.0 \%, p=0.08)$, although there was no significant difference. There was no difference in the use of aspirin or angiotensin converting enzyme inhibitors between the two groups.

In-hospital mortality was significantly higher in women compared to men $(24.0 \% v 13.9 \%, \mathrm{p}=0.02)$ (fig 1$)$. Women were more likely than men to develop stroke $(2.9 \% v 1.0 \%)$ and heart block $(6.4 \% \vee 3.3 \%)$, although these differences were not significant. There was no significant difference in the incidence of bleeding complications. The mortality rate was significantly lower among patients who received thrombolytic treatment $(9.1 \%)$. Although on univariate analysis being female was a predictor of increased risk of in-hospital mortality (odds ratio (OR) 1.139, 95\% confidence interval (CI) 1.076 to $1.206 ; \mathrm{p}=0.0001$ ) after adjustment for all baseline differences, female sex was not an independent predictor of increased in-hospital mortality (OR 1.03, $95 \%$ CI 0.89 to $1.27 ; \mathrm{p}=0.754$ ).

\section{DISCUSSION}

The current study on a Middle Eastern population demonstrates higher in-hospital mortality in women than in men after AMI. This higher risk is related to unfavourable baseline clinical characteristics including older age, higher incidence of diabetes, hypertension, and hypercholesterolaemia, and less likelihood of receiving thrombolytic treatment.

Several observational studies reported higher in-hospital mortality after AMI in women when compared to men. ${ }^{12}$ This difference was in part because women were older and more likely to have co-morbid conditions such as diabetes mellitus. However, in other studies, the mortality rate reported is similar. ${ }^{3}$ Vaccarino and colleagues recently 
demonstrated a significantly higher in-hospital mortality rate in women compared to men $(16.7 \% v 11.5 \%){ }^{2}$ Moreover, among patients above 50 years of age, the mortality rate for women was more than twice that for men; with increasing age this difference disappeared. Young women were more likely than young men to have diabetes, and a history of congestive heart failure and stroke, but no sex based differences were apparent at older ages.

Data on the outcome of women after AMI are limited to the western population and to the best of our knowledge are limited to two studies in other communities. ${ }^{45}$ Pimenta and colleagues studied 600 consecutive patients (435 men and 165 women) with AMI admitted to a hospital in Brazil and demonstrated higher mortality in women compared to men $(23 \% v 9.9 \%, p=0.02) .{ }^{4}$ Furthermore, being female was an independent predictor of mortality (OR 2.73, p = 0.001). Hapaz and colleagues evaluated the ethnic effect on survival after AMI in 5692 patients and demonstrated a higher inhospital and long term (7.1 (3.5) years) mortality rate in women than in men $(57.6 \% v 43.3 \%){ }^{5}$ In addition, the risk ratio differed significantly in women based on ethnic origin; lower in Jewish women born in central Europe compared with counterparts born in the Middle East.

In conclusion, this study extends the observations of previous sex studies in an ethnic population that has not previously been studied, and is consistent with those studies in demonstrating higher co-morbid conditions and worse outcome in women compared to men. This worse outcome is explained by unfavourable baseline clinical characteristics and a lesser likelihood to be treated aggressively with medications that have been conclusively shown to improve outcome. Regional and global measures to fight coronary artery disease in women are urgently needed.

\section{Authors' affiliations}

J Al Suwaidi, H A Al Binali, Department of Cardiology and

Cardiovascular Surgery, Hamad General Hospital and Hamad Medical Corporation, Doha, State of Qatar

A Bener, Department of Medical Statistics, Hamad General Hospital and Hamad Medical Corporation

S Behair, Section of Health Education, Hamad General Hospital and Hamad Medical Corporation

Correspondence to: Dr J Al Suwaidi, Department of Cardiology and Cardiovascular Surgery, Hamad Medical Corporation and Hamad General Hospital, PO Box 3050, Doha, State of Qatar;

jha01@hmc.org.qa

Accepted 27 October 2003

\section{REFERENCES}

1 Jneid H, Thacker HL. Coronary artery disease in women: different, often under-treated. Cleve Clin J Med 2001 May;68:441-8.

2 Vaccarino V, Parsons L, Every NR, et al for the National Registry of Myocardial Infarction 2 Participants. Sex-based differences in early mortality after myocardial infarction. N Engl J Med 1999;341:217-25.

3 Pohjola S, Siltnen P, Romo M. Five-year survival of 728 patients after myocardial infarction. Br Heart J 1980;43:176-83.

4 Harpaz $D$. Ethnic differences in mortality of male and female patients surviving acute myocardial infarction: long-term follow-up of 5,700 patients. The secondary prevention reinfarction israeli nifedipine trial (SPRINT) study group Eur J Epidemiol 1997 Oct;13:745-54.

5 Pimenta L, Bassan R, Potsch A, et al. Is female sex an independent predictor of in-hospital mortality in acute myocardial infarction? Arq Bras Cardiol $2001 ; 77: 37-50$.

\section{IMAGES IN CARDIOLOGY}

\section{Ventricular aneurysm secondary to sarcoid disease}

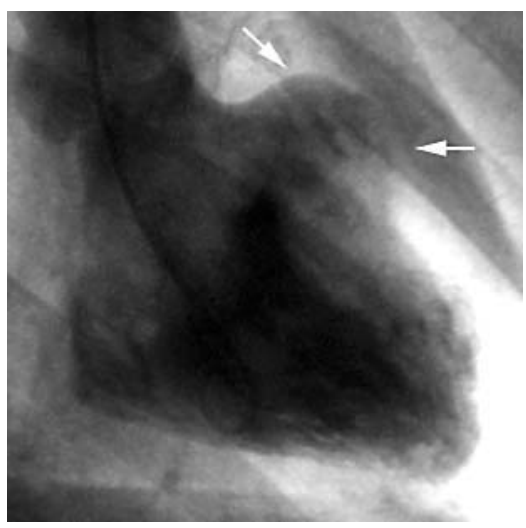

$\Lambda$

58 year old man, previously diagnosed with sarcoidosis, presented with acute onset palpitations. ECGs showed a broad complex tachycardia with a downward axis and a heart rate of 160 beats per minute, requiring cardioversion. Subsequent coronary angiography showed normal coronary arteries. The left ventriculogram illustrated a discrete saccular aneurysm on the anterior wall of the left ventricle. Endocardial biopsy showed evidence of non-caseating granulomata consistent with sarcoidosis. Staining for acid-fast bacilli and fungi were negative. The patient received an implantable cardioverter-defibrillator, and steroids. At follow up he is doing very well and enjoying an almost normal life.

Clinically evident cardiac involvement in sarcoidosis is rare. Historically most cases are reported post-mortem. The majority of reports are of heart failure and arrhythmias. Diagnosis depends on a suggestive clinical picture combined with appropriate histology from some organ, and the exclusion of other causes of non-caseating granulomata. When we tried to investigate the best management of cardiac sarcoid and in particular ventricular aneurysms, we found that there was a paucity of evidence available. The accepted practice appears to be instigation of corticosteroids, plus any appropriate additional cardiac intervention in the form of pacemakers and medication. In conclusion, we illustrate an interesting but unusual presentation of cardiac sarcoid.

A Marks

M H Anderson

N K Harrison

Angharad.marks@talk21.com 